www.jmscr.igmpublication.org

Index Copernicus Value: 79.54

ISSN (e)-2347-176x ISSN (p) 2455-0450

crossrefDOI: https://dx.doi.org/10.18535/jmscr/v7i1.204

\title{
A Rare Case of Isolated Inguinal Lymph Node Metastasis from Breast Carcinoma - Case Report
}

\author{
Authors \\ Yalavarthi Pavan Ganesh ${ }^{1}$, Arulappan.T ${ }^{2}$, Sivaraja ${ }^{3}$, Kishan .M ${ }^{4}$ \\ ${ }^{1,2,3}$ Dept of General Surgery, Sri Ramachandra Institute For Higher Education and Research, Chennai, India

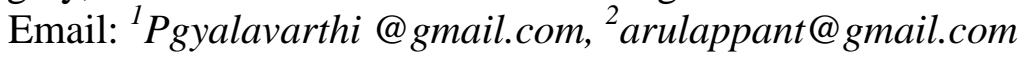

\begin{abstract}
Surgery and irradiation for breast cancer may interfere with conventional pathways of spread, leading to bizarre patterns of dissemination through lymphatics or through hematogenous route. We report on a case of a premenopausal woman with breast cancer, the primary tumor was T3N1M0, case of triple negative breast cancer, After mastectomy the patient received adjuvant chemotherapy. After one year patient came with complaints of left inguinal pain followed by swelling.and unusual disease dissemination with inguinal lymph node metastasis.
\end{abstract}

Keywords: Breast cancer, Inguinal node metastases; Lymphatic pathways.

\section{Introduction}

As it is common knowledge, breast cancer tends to metastasize through both lymphatic pathways to regional nodes as well and hematogenous spread to organs such as the liver, lung, bones, brain, etc. However, isolated involvement of distant nodal regions is extremely uncommon. We present a case of triple negative metachronous breast cancer with generalized neurofibromatosis that developed isolated inguinal node metastases.

\section{Case Report}

33 year old premenopausal female patient who was a treated case of Locally Advanced Breast Cancer T3N1M0 came with complaints of left inguinal pain followed by swelling for three months. The pain was throbbing in nature, limiting her mobility and was not relieved on rest. No history of fever / vomiting/any other swelling in the body/ back pain /abdominal distension/ headache /blurring of vision/ breathlessness /cough/ haemoptysis/ jaundice.

On physical examination there was single left inguinal node $1 \times 2 \mathrm{~cm}$ firm, mobile, tender, smooth, well defined borders, skin over the swelling normal. Patient is a known case of generalised neurofibromatosis for 15 years. One year back patient came with complaints of lump in the left breast for three months and diagnosed to have carcinoma of left breast Staging work up with Bone scan, USG abdomen, Chest Xray, /Brain MRI showed no evidence of metastasis. and was diagnosed to have locally advanced breast cancer (T3N1M0) Patient treated with 5 neoadjuvant cycles of adriamycin and cyclophosphamide followed byleft Modified radical mastectomy and 3 adjuvant cycles of placitaxel.

Histopathology showed Invasive Lobular carcinoma pT3N1M0, ER PR Negative, HER2 NEU Negative, KI $67-40 \%$. Post treatment patient was 


\section{JMSCR Vol||07||Issue||01||Page 1167-1170||January}

asymptomatic for one year. FNAC done and it revealed Metastatic carcinomatous deposit in left inguinal node. Excision Biopsy of left inguinal node showed metastatic carcinomatous deposit. PET Scan done which revealed multiple bony metastasis and inguinal metastatic lymph nodes and was proceeded with chemotherapy.

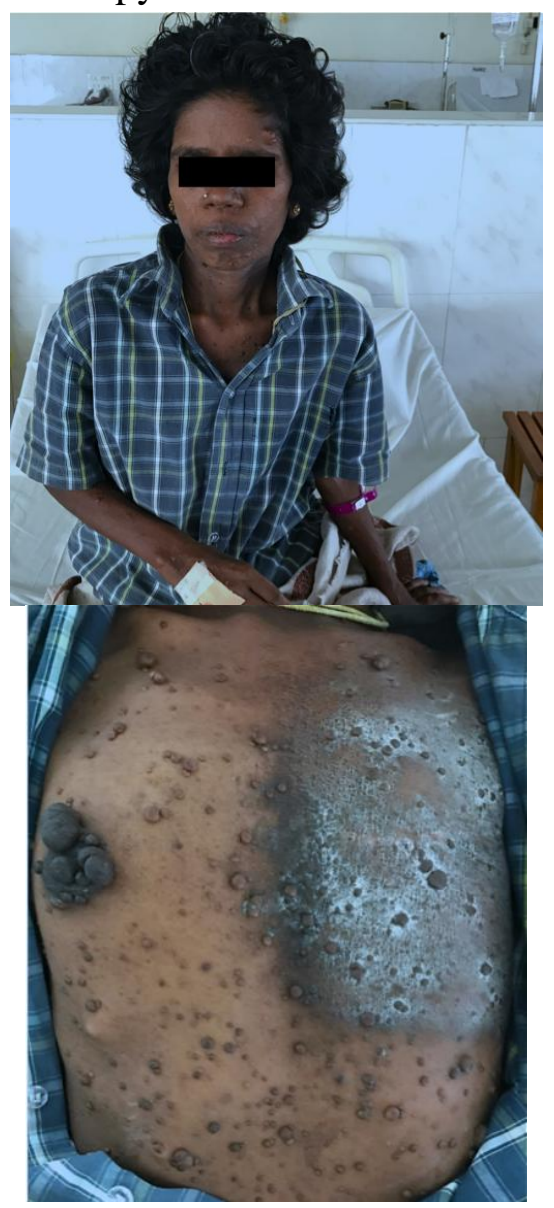

Figure 1: Picture showing left modified radical mastectomy with generalized neurofibromatosis.

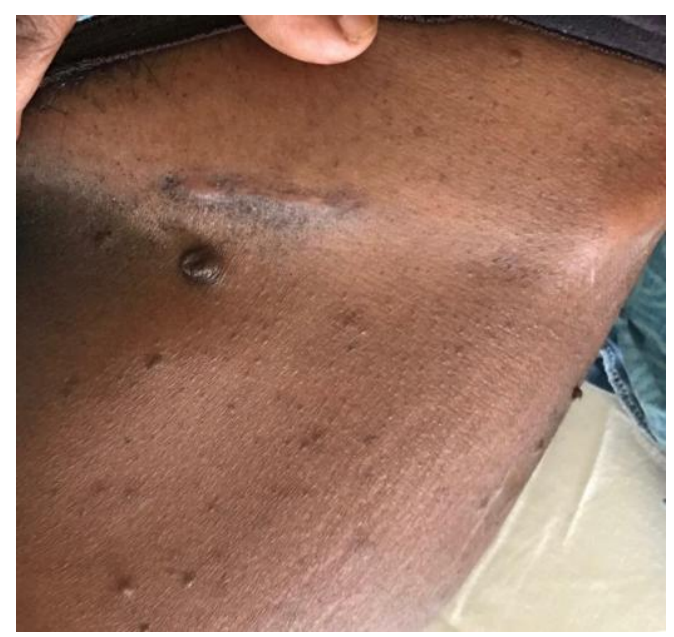

Figure 2: Post excision biopsy of left inguinal node.

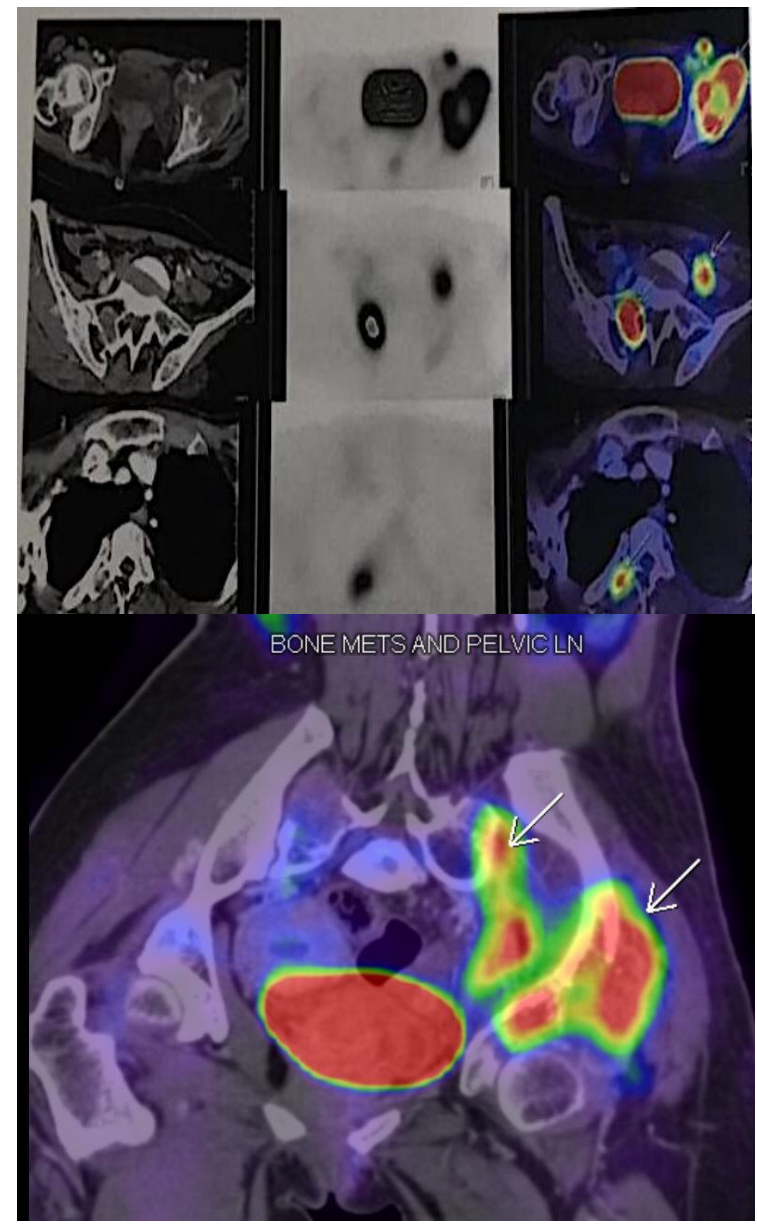

Figure 3: PET scan showing sclerotic bony lesion with increased metabolic activity and lymph node involvement.

\section{Discussion}

Breast cancer is currently the fifth most common cause of cancer death worldwide after lung, stomach, liver and colon. It represented a high proportion $(11.3 \%)$ of all primary malignancies and generated a large number of metastases (5.2 per primary malignancy; As a result, breast cancer contributed $23.6 \%$ of all metastases the highest number among all analyzed malignancies. ${ }^{(1)}$

The initial sites of locoregional spread include frequently the axillary nodes, moderately the internal mammary nodes and rarely the lymph nodes near the intercostal arteries and veins. Metastatic sites involved accounts for lymph node regional lymph node $12.2 \%$, (distant) $10.2 \%$, lungs $11 \%$, liver $9.8 \%$, pleura $7 \%$, adrenal $7 \%$, skin $5.7 \%{ }^{(1)}$

Hence the most common site of nodal metastasis is the axillary region followed by the supraclavicular 
region. Isolated inguinal lymph node metastasis as clinical manifestation of breast carcinoma is quite infrequent and rarely reported in the literature.

The first such report by Baba et al. described a patient with inguinal node and breast lump at presentation $^{[2]}$. Appearance of the inguinal node preceded the breast lump by a year. Other metastatic workup was negative. Kilc iksiz et al described a patient with early breast carcinoma (T2N0M0, triple negative, lymphovascular space invasion) treated with breast conservation surgery, adjuvant RT and chemotherapy .Five years postoperatively, she developed right leg edema and pain. Imaging done for persistent complaints which showed right inguinal node $(3 \mathrm{~cm})$ along with right iliac and retroperitoneal nodes. Workup for other abdominal malignancies was negative. Biopsy from inguinal node revealed triple negative microtubular adenocarcinoma. ${ }^{[3]}$

Jebbin et al have described two more cases that presented with inguinal node metastases Retrograde embolization was suggested as the probable pathogenesis. They postulated that in the presence of altered lymphatic pathways after surgery or irradiation, such involvement should be regarded as regional recurrence and considered for radical therapy. ${ }^{(4)}$

A condition that has been described in under 15 cases till date is the presence of ectopic breast tissue in the vulva with inguinal nodal involvement could be the other possible etiology. In the presence of nodal metastases, obstruction of the physiologic routes of lymphatic flow may occur and alternative pathways may then become important. But in early breast cancer these alternative pathways are not well established $^{[5]}$.

The alternative routes that have been described are deep, substernal drainage to the contralateral internal mammary chain, superficial presternal crossover, lateral intercostal and mediastinal drainage, and spread through the rectus abdominis muscle sheath to the subdiaphragmatic and subperitoneal plexus (Gerota's pathway) ${ }^{[6]}$

Except extrahepatic and extraskeletal metastases in the abdominal region, the lymphatic system is the most frequently involved system following the gastrointestinal system. Retroperitoneal and mesenteric lymph nodes were the mainly involved nodes. Isolated intraabdominal or inguinal lymph node metastases were reported only rarely in various patient series, Therefore it is difficult to define the frequency of abdominal lymphatic involvement. $^{(7)}$

The initial tumor type may yield clues about the sites of metastasis. Infiltrating ductal carcinoma (IDC) commonly involves the lungs, pleura and brain, whereas infiltrating lobular carcinoma (ILC) most commonly involves the bone marrow, peritoneum and retroperitoneal structures, such as the ureters . Our case was diagnosed with ILC with the histology of both primary and metastatic tumor $^{(8)}$. Hormone receptors (ER, PR) in tumor cells is a recommended method to know about the breast origin of metastases. In our case, the metastatic tumor was ER and PR negative.

First, a selected subset of metastases patients should be approached with curative, not palliative, intent. Second, the current minimalistic postoperative monitoring should be revised to allow early diagnosis of low-burden disease relapse. Several trials have tested the hypothesis that high-dose chemo- therapy (HDC) with autologous bone marrow or peripheral stem cell transplantation may improve survival in women with $\mathrm{MBC}$ (metastatic breast carcinoma) ${ }^{(9)}$

Gene expression profiling may identify tumors that are likely to metastasize, even the location of the metastases, and may identify predictive markers of sensitivity to different therapies. As a result, each patient would be offered an individualized treatment associated with the highest probability of therapeutic benefit and the smallest risk of resistance ${ }^{(10)}$. Therapeutic developments, such as the introduction of new cytotoxic agents (taxanes, vinorelbine, capecitabine, gemcitabine, etc), thirdgeneration aromatase inhibitors and fulvestrant, biological therapies (trastuzumab, lapatinib, and bevacizumab), have resulted in constant improvements in treatment efficacy and consequently in metastatic outcome $e^{(11)}$ 


\section{Conclusion}

Altered lymphatic pathways could have been responsible for the inguinal involvement, but without doubt, inguinal involvement was associated with early systemic progression. hence a direct hematogenous spread is also possible. As the number of cases with unexpected sites of tumor dissemination increase, clinicians should maintain a high degree of suspicion when coming across with unusual complaints and findings.

\section{References}

1. Metastatic Patterns of Cancers-diSibio \& French Arch Pathol Lab Med. 2008; 132:931-939.

2. Baba M, Tatsuta M, Miya A, Ishida $H$, Masutani S, Kawasaki T, et al. A case of breast cancer diagnosed by inguinal lymph node metastasis. Breast Cancer 2000;7:1735.

3. Kilc, iksiz S, Go“kc, e T, Kinay M. Isolated inguinal lymph node metastasis from breast carcinoma - case report and review of the literature. J BUON 2006;11:229-32.

4. Jebbin NJ, Adotey JM. Metastatic carcinoma of the breast with inguinal lymph node involvement: a report of two cases.Niger $\mathbf{J}$ Clin Pract 2008;11:383-5.

5. Sood A, Youssef IM, HeIba SI et al. Alternative lymphatic pathway after previous axillary node dissection in recurrent/ primary breast cancer. Clin Nucl Med 2004; 29: 698-702.

6. Isolated inguinal lymph node metastasis from breast carcinoma - case report andreview of the literature S. Kilçiksiz, T. Gökçe, M. Kinay Journal of BUON 11: 229232, 2006

7. Valenzano M, Papadia A, Lorenzi P et al. Breast cancer metastatic to vulva after local recurrence occurring on a rectus abdominis myocutaneous flap: a case report and review of the literature. Eur J Gynaecol Oncol 2003; 24: 577-579.

8. Jain S, Fisher C, Smith P et al. Patterns of metastatic breast cancer in relation to histological type. Eur J Cancer 1993;29: 2155-2157.

9. Farquhar C, Marjoribanks J, Basser R, et al. High dose chemotherapy and autologous bone marrow or stem cell transplantation versus conventional chemotherapy for women with metastatic breast cancer. Cochrane Database Syst Rev. 2005;(3): CD003142. .

10. Andreopoulou E, Hortobagyi GN. Prognostic factors in metastatic breast cancer: successes and challenges toward individualized therapy. $J$ ClinOncol. 2008;26(22):3660-3662.

11. International Guidelines for Management of Metastatic Breast Cancer: Can Metastatic Breast Cancer Be Cured? Olivia Pagani, Elżbieta Senkus, William Wood, Marco Colleoni, Tanja Cufer, Stella Kyriakides, Alberto Costa, Eric P. Winer, Fatima Cardoso, on behalf of the ESO-MBC Task Force J Natl Cancer Inst 2010;102:456-463 